\title{
Dysregulation of Phospholipid Metabolism in Synaptic Membranes and Their Role in Encephalopathy Forming After a Hemorrhagic Shock
}

\author{
Galina Fedorovna Leskova \\ Laboratory of nanopathology, nanotoxicology and biomedical nanotechnologies, Institute of General Pathology und Pathophysiology, \\ Moscow, Russia
}

Email address:

leskovagalina@rambler.ru

To cite this article:

Galina Fedorovna Leskova. Dysregulation of Phospholipid Metabolism in Synaptic Membranes and Their Role in Encephalopathy Forming After a Hemorrhagic Shock. American Journal of BioScience. Vol. 3, No. 4, 2015, pp. 133-140. doi: 10.11648/j.ajbio.20150304.13

\begin{abstract}
Phospholipids (PLs) of neuronal membranes are active universal neuromodulators. They regulate many functions of the neurons, including receptors signaling, which during a hemorrhagic shock (HS) get damaged, leading to encephalopathy. An analysis of the data, presented in this review, suggests that the dysregulation of PL metabolism in synaptic membranes is a key mechanism of encephalopathy during HS. Stabilizing the PL composition of the neuronal membranes may become one of the most important treatment methods for shock-induced disorders of brain functions.
\end{abstract}

Keywords: Phospholipids, Synaptic Membranes, Hemorrhagic Shock

\section{Introduction}

High vulnerability of the central nervous system at shock genicity exposures defines difficulty of restoration of its functions at HS. One of the important CNS damage elements in the post-resuscitation period of HS is the phenomenon of non-reinstatement of synaptic contacts between neurons, which occurs as a result of changes in the structure of the preand postsynaptic membranes [1]. Changes in receptor systems, processes of signal generation and transduction in the brain tissue during circulatory disorders and in the post-resuscitative period are the leading pathogenic components of post-ischemic encephalopathy [2]. It is known that brain activity is largely determined by the metabolism of PLs of neuronal membranes, which are active universal neuromodulators. Activation of phospholipases leads to the modification of the lipid bilayer of nerve endings. Metabolites of neuronal synaptic membranes PLs efficiently activate many membrane-associated enzymes, as well as, endocytosis and exocytosis of neurotransmitters and control pathogenic mechanisms of a number of neurodegenerative diseases [3, 4]. Disruption of membrane PLs significantly contributes to neuronal death at pathological conditions [5]. Based on the foregoing, research of PL metabolism features in synaptic membranes in various parts of the CNS seems promising in the development of new approaches to the treatment of HS.
In this review we analyzed the mechanisms of PL metabolic disorders influence in synaptic membranes on processes of transmission in the frontal areas of the brain and medulla oblongata. In the frontal part of the brain is located the associative area of the cortex. The importance of studying the disorder mechanisms of compensatory ability of the cells of the medulla oblongata during HS is associated with the participation of the medulla oblongata structures in the regulation of vascular tone and coordination of blood circulation and respiration, as well with the regulation of activity of highest brain departments.

In the medulla oblongata, there are nerve cells that contain neurotransmitters such as acetylcholine, catecholamines, serotonin, neuropeptides (including met- and leu-encephalin, substance $\mathrm{P}$, somatostatin, neurotensin, cholecystokinin, vasoactive intestinal peptide, pancreatic polypeptide) [6], which demonstrate the complexity of regulating this part of the CNS.

\section{The Role of Phosphatidylinositol in Compensatory and Pathological Changes of Neurotransmission}

The general tendency of the phospholipids composition changes in synaptic membranes of the medulla oblongata and 
frontal region of the brain during the early stages of HS is the reduction of phosphatidylinositol (PI) level [7, 8]. The metabolism of inositol-containing PLs is considered as the general transduction mechanism for extracellular signals, mediated by various biologically active substances, including neurotransmitters. The interaction of mediators, released from the presynaptic membrane, with the receptors of the postsynaptic membrane, forms the active receptor-ligand complex, which initiates neurotransmission along the way defined by the phosphorylated form of the PI. Considering that the activation of cholino- and adrenoceptors is a factor leading to the stimulation of phosphoinositide hydrolysis [9. 10], we can deduce that adrenergic and cholinergic hyperactivity, induced by massive blood loss, leads to an accumulation of PI decay product inositol-1,4,5-trisphosphate, - capable to activating the $\mathrm{Ca}^{2+}$ channel for take $\mathrm{Ca}^{2+}$ from the extracellular space [11]. Since the hydrolysis of PI-4-phosphate and PI 4, 5-bisphosphate can occur at concentrations of $\mathrm{Ca}^{2+}$ in the cytoplasm of cells corresponding to the state of rest (about $10^{-7}$ ), while the hydrolysis of PI by phospholipase $\mathrm{C}$ requires higher concentrations of $\mathrm{Ca}^{2+}$, the initial increase of phosphoinositide hydrolysis, as result of massive hemorrhage, is necessary for the subsequent activation of PI hydrolysis.

It has been shown that $\mathrm{Ca}^{2+}$-binding protein- neuronal calcium sensor-1 (NCS-1) - is presented in synaptic membranes and in the membranes of synaptic vesicles and participates in processes of neurotransmission [12, 13]. Against the background of activation of phospholipase $\mathrm{C}$ NCS-1 increases the agonist-induced and $\mathrm{Ca}^{2+}$-mediated transmembrane signaling. It is believed that either cytoplasmic NCS-1 or NCS-1 of vesicle membrane binds to PI 4-kinase, that is located on the plasma membrane and catalyzes the initial step of PI phosphorylation, by increasing enzyme activity. As consequence, there is an increase in formation PI 4-phosphate - a metabolite, of which PI 4, 5-bisphosphate later is formed - major substrate for PI-specific phospholipase $\mathrm{C}$. The regulatory role of hydrophobic diglycerides released from PI should also be noted as they remain in the membrane and at presence phosphatidylserine (PS) activate protein kinase C, which controls the synthesis and secretion of neurotransmitters and changes in receptor sensitivity [14]. The activation of PI-specific phospholipase $\mathrm{C}$ may be induced by the stimulation of metabotropic glutamate receptors and such activation depends on the availability of extracellular $\mathrm{Ca}^{2+}$ [15]. In this aspect it is interesting to consider the hypothesis about the protective role of metabotropic glutamate receptors, considering that the activation of protein kinase $\mathrm{C}$ caused by stimulation of these receptors, leads to the phosphorylation (desensitization) of ionotropic NMDA-receptor [16], which play a leading role in the induction of ischemic damages due to their high affinity for excitatory amino acids (EAAs) and high permeability for $\mathrm{Ca}^{2+}$ [17]. The stimulation of phospholipase $\mathrm{C}$, initiated by neurotransmitters, is regulated by GTP-activated by G-protein, which links the receptor stimulus with the signaling pathway inside the cell.
G-protein-coupled receptor-mediated signaling is attenuated at activation of the processes, directed on desensitization. In these processes the initial role is played by the agonist-dependent phosphorylation of specific G-protein-coupled kinases, including protein kinases A and C $[18,19]$. The stimulation of these kinases disturb coupling of the receptors with G-proteins, thereby attenuating the activating effect of agonists on the receptor-mediated activation of phospholipases, thus limiting the magnitude and duration of receptor signaling. There is also a model in which the activation of protein kinase $\mathrm{C}$ leads to an increase of phosphorylation of the protein that transfers PI from places of its intracellular synthesis into the plasmalemma, depriving this protein of transport function and thus being responsible for the inhibition of receptor signaling [20]. It is believed that when protein kinase $\mathrm{C}$ impairs the delivery of PI into the cell membrane, it reduces phosphoinositide synthesis and consequently, reduces phospholipase C-mediated signaling.

The mechanisms of PI metabolites participation in regulation of the neurotransmission processes is of interest. In particular, the importance of PI 4, 5-bisphosphate in the regulation of signal transmission is defined by controlling of the reconstruction of protein-targets, which mediate the membrane transport (including endocytosis, exocytosis and binding vesicles), during its interaction with phosphoinositide-binding domains of these proteins [4, 20]. PI-monophosphate also possesses fusogenic activity, since a change in its concentration in the acceptor membrane has an effect on the physical and chemical properties of the receptor environment and thus regulates their interaction with mediator vesicles [21]. The fusogenicity of PI 4-phosphate and PI 4, 5-bisphosphate undoubtedly increases the affinity of the receptors towards ligands in the frontal part of the brain and medulla oblongata on the early stages of HS. Furthermore, PI-4, 5-bisphosphate inhibited the ability of $\beta$-adrenergic receptor kinases 1 and 2 to phosphorylation (desensitization) of agonist-occupied $\mathrm{m} 2$ muscarinic acetylcholine receptors [22], what may be one of cholinergic hyperactivity mechanisms during the development of HS. It is known that the stimulation of the synthesis of PI 4-phosphate is connected with a $\mathrm{Ca}^{2+}$-mediated increase in release of noradrenaline and glutamate [13]. Thus consider, that the activation of PI-4-kinase, providing a substrate for the synthesis of PI 4,5-bisphosphate, mediates the opening of voltage-dependent $\mathrm{Ca}^{2+}$ channels of $\mathrm{P} / \mathrm{Q}$ and $\mathrm{N}$-types, which regulate the exocytosis of neurotransmitters in nerve endings. Furthermore, inositol 1, 4, 5-trisphosphate, plays a large role in signal transduction, promoting increase in neurotransmitter release from nerve endings through $\mathrm{Ca}^{2+}$-dependent pathway [11]. The inositol 1, 4, 5-triphosphate-dependent pathway of mediators exocytosis increase is described, in particular, for the mechanism of activation of muscarinic cholinergic receptors [23].

A boost of PI catabolism may be caused by influence of PI 3-kinase, which plays a crucial role in the regulation of systemic blood pressure. Increased expression of PI 3-kinase in brain leads to an increased adrenergic stimulation induced 
by angiotensin II [24]. PI 3, 4, 5-trisphosphate and PI 3, 4-bisphosphate are key components of the signaling pathway which prevents apoptosis.

There are at least three distinct PI-specific phospholipase D isozymes, which control the breaking down of PI 3, 4, 5-trisphosphate up to inositol 3, 4, 5-trisphosphate [25]. These PI-specific phospholipases D are $\mathrm{Ca}^{2+}$-dependent. It is believed that the activation of PI-specific phospholipase D promotes a transient increase in the content of phosphatidic acid (PA), by a way, different from way of phosphatidylcholine (PC)-specific phospholipase D. In this connection, the role of PA in enhancing the formation of superoxides through the stimulation of NADPH-oxidase becomes interesting [26]. It is possible, however, to believe that the activity of superoxides in the synaptic membranes of the medulla oblongata, in the early stage of development of $\mathrm{HS}$, in a certain way, is limited due to the high content in these membranes of PC [7] possessing the antioxidant properties [27]. It is generally believed that the activation of PI-specific phospholipase D is a risk factor of pathological conditions. On the other hand, 3-phosphatase dephosphorylates PI 3, 4, 5-trisphosphate up to a secondary messenger - PI 4, 5-bisphosphate, which undoubtedly promotes neurotransmission increase.

Decrease of PI level in synaptic membranes on early stage of HS may be caused by activation of phospholipase A2, which is active both with an increase in the concentration of $\mathrm{Ca}^{2+}$ and in its absence under the condition that PI metabolites are present [28]. The activation of both isoforms of phospholipase $\mathrm{A}_{2}$ is partly the mechanism mediating the change of properties of AMPA-sensitive glutamate receptors, playing an important role in the modulation of neurotransmission $[29,30]$. It is possible also to note the role of phospholipase $A_{2}$ in enhancing the secretion of neurotransmitters in the nervous system, mediated by independent of $\mathrm{Ca}^{2+}$ liberation of fatty acids (especially arachidonic) from the PLs of the synaptic membranes, which initiates the fusion of the mediator vesicles with the acceptor [31]. It is believed that phospholipase $\mathrm{A}_{2}$ plays a crucial role in cell damage in CNS, given its importance in the preferable hydrolysis of PLs, containing arachidonic acid, which has both physiological and pathophysiological importance. Considering the involvement of PI metabolites - PI 3, 4, 5-trisphosphate and PI 4, 5-bisphosphate - in the signaling mechanisms that prevent the development of apoptosis [25], it can be expected that the formation of phosphorylated derivatives of PI in combination with the release of arachidonic acid concerns mechanisms, promoting the development of inflammatory processes in the neurons of the medulla oblongata during HS. Furthermore, it is known that arachidonic acid is a regulator of cellular processes such as the activation of protein kinase $\mathrm{C}$ and the modulation of ion channels activity [28]. Arachidonic acid stimulates NMDA-receptors and enhances glutamate concentration in the synaptic cleft through increase in its release and/or through reuptake blockade $[32,33]$. It can directly affect the integrity of the membrane, acting as a detergent. Thus, the initial reduction of PI level in the synaptic membranes of the frontal part of the brain and the medulla oblongata during the initial stage of HS [7, 8] may be associated with an increased secretion of neurotransmitters - noradrenaline and acetylcholine, - induced by blood loss and may be reflexion of the existence of the of critical cellular mechanisms which control the modulation of the excitability of neurons and are ways to maintenance of their viability. Moreover, adrenergic and cholinergic hyperactivity of the CNS can be considered as one of the mechanisms of receptors stimulation of EAAs, what is consistent with the existing ideas about the significance in the regulation of these receptors work of adrenergic and cholinergic influences [34, 35]. The activation of metabotropic glutamate receptors, apparently, significantly contributes to enhancing compensatory mechanisms of neurotransmission. It is possible that an increased metabolism of PI of the synaptic membranes is a factor limiting the impact of the excitatory neurotransmitters on the neurons and represents one of the protection key features of neurotransmission pathways in the frontal part of the brain and medulla oblongata during a developing HS. A relative weakening of the metabolism of PI in the synaptic membranes of the medulla oblongata is observed in later stages of HS [7]. It is possible that this fact is partly due to the activation of ionotropic glutamate receptors, since this activation causes inhibition of various metabotropic receptors, including glutamate and muscarinic receptors which mediated phospholipase $\mathrm{C}$ activation [36]. A characteristic feature of the changes in the composition of PLs in the synaptic membranes of the frontal part of the brain during the late stage of $\mathrm{HS}$ is the considerable increase in the concentration of PI [7] that specifies in a significant inhibition in the activity of phospholipase C. In connexion with this, data about the delayed post-ischemic regional decrease in phospholipase $\mathrm{C}$ expression in the brain are of interest [37]. Authors of this research connect this fact with definitive degradation of dendrites.

\section{Dysregulation of Phosphatidylcholine Metabolism and Weakening of Neurotransmission}

The depletion of PC may be considered as one of the main factors damaging synaptic membranes of the medulla oblongata that can be observed in the late stages of HS [7]. A range of mechanisms of the PC hydrolysis modulation independent on the activity of protein kinase $\mathrm{C}$ and conversely dependent on it, - have been described. In particular, it is known that during cholinergic hyperactivity and the increased demand in the acetylcholine precursor choline - the stimulation of PC- specific phospholipase D, controlling the formation mechanism of acetylcholine from PC in the cell membranes of CNS, becomes important [38]. There is also an activation mechanism for this enzyme, connected with stimulation of $\alpha 1$-adrenergic receptors [39]. Furthermore, the role of glutamate in the activation of 
PC-specific phospholipases $\mathrm{A}_{2}, \mathrm{C}$ and $\mathrm{D}$ has been described [40]. Protein kinase $C$ is characterised by dual influence on the catalytic activity of phospholipase D. On the one hand, protein kinase $\mathrm{C}$ stimulates phospholipase D (the path that does not require ATP), and on the other hand - the phosphorylation by protein kinase $\mathrm{C}$ of this enzyme correlates with its inhibition [41, 42]. Taking into consideration the energy deficit, typical for later stage of HS, the dominance of the stimulating effect of protein kinase $\mathrm{C}$ on the PC metabolism in the synaptic membranes of the medulla oblongata can be expected for this period.

A fall in the level of PC in the synaptic membranes of the frontal part of the brain occurs in the early stages of the HS [8] and is combined with a decrease in the concentration of PI. In connexion with this are of interest the observations, that during increase of the neurotoxic processes the increase in the hydrolysis of PI is an initial step of $\mathrm{Ca}_{2}^{+}$-dependent processes, which lead to the damage of the structure of the neurons [4]. It was found that the mobilization of the PI-dependent signaling pathway is often accompanied by the activation of PC-specific phospholipase D [44]. The metabolite of the PI - PI 4, 5-bisphosphate - is a special activator of this enzyme. On the other hand, it should be noted that activation of the PC-specific phospholipase D increases the release of PA from PC. Observations on the ability of PI 4, 5-bisphosphate to activate $\mathrm{PC}$-specific phospholipase D and the data on the participation of $\mathrm{PC}$ in the activation of PI 4-phosphate kinase formed the basis of the model in which the formation of PA and PI 4, 5-bisphosphate take part in the circuit, which plays an important role in fusion of vesicles with acceptor membranes [45]. It is believed that the interaction of the mediators with acceptors of membranes activates the brain membrane phospholipase D. This leads to the release of PA from PC, accompanied by an increased activity of PI 4-phosphate 5-kinase, presumably localized on the acceptor membrane. This fact is considered as the cause for the increase in the formation of PI 4, 5-bisphosphate, which activate the PC-specific phospholipase D. The metabolic pathway described can cause very rapid and profound changes in the lipid composition of the membranes, accompanied by the formation of micro domains depleted in PI and later on - in PC. At a late stage of HS, the PC level in the synaptic membranes of the frontal part of the brain are normalized [8]. This circumstance may be the result of an increased energy deficit, that prevent the phosphorylation of PI. The foregoing suggests that membrane PI metabolism plays a key role in the regulation of the functional activity of the frontal part of the brain.

The high biochemical activity of PC metabolites allows to consider the decrease of this PL level in synaptic membranes among the key factors that trigger the weakening of neurotransmission in the brain during HS. In this connexion PC seems to play an important role in the stimulation of superoxide formation [26]. Given that PC is the main PL in cell membranes, the enhanced damaging effects of another metabolite PC - arachidonic acid - can be expected. It should be noted that arachidonic acid and diglycerides released from the PC are activators of protein kinase C [28].

It is known that stimulation of NMDA-receptors, mediating the release of choline from PC; prevent the inclusion of this metabolite back to the PC, inhibiting the activity of choline phosphotransferase. This path of glutamatergic activation precedes death of neuronal cell [46]. Therefore, it is possible that in late stages of HS in the synaptic membranes of the medulla oblongata may be a disturbance of PC synthesis through the CDP-choline formation - metabolic pathway, which is fundamental in the synthesis of PC in the brain [47]. Depletion of PC in synaptic membranes can be seen as a increase factor of nonspecific permeability of the neurons, mediating their swelling. Medulla oblongata is subjected to the greatest damaging action.

\section{Dysregulation of the Phosphatidylethanolamine Metabolism and Excitotoxicity}

In the synaptic membranes of neurons the metabolic pathway of phosphatidylethanolamine (PE) has an important physiological significance. It is controlled by $\mathrm{N}$-methydyltransferase and is related to the synthesis of PC, participating in the formation of the acetylcholine precursor choline. Increased level of PE, and low content of PC are a distinctive feature of the changes in the PLs spectrum of the synaptic membranes of the frontal part of the brain and medulla oblongata during the development of HS [7, 8]. This circumstance does not exclude possibility of slowing down the process of methylation of PE in the nerve endings. This may be due to the accumulation of cytoplasmic $\mathrm{Ca}^{2+}$, which observed in neurons after massive blood loss [48]. $\mathrm{Ca}^{2+}$ inhibits the activity of phosphatidylethanolamine-specific $\mathrm{N}$-methyltransferase [49], that may be accompanied by a further complication in the synthese of PC. $\mathrm{N}$-methyltransferase has a specific role in regulating the activity of nerve cells. In particular, the methylation of PLs is considered as an important mechanism for the transmission of nerve impulses, mediated through amino acids [50] using which, nerve cells regulate the concentration of amino acids in neurons. The high level of methylation of PLs in the synaptic membranes is accompanied by a decrease in the capture of EAAs by the nerve endings, while at low level of PLs methylation the inhibition of EAA capture is decreased [50]. Therefore, the PE accumulation in synaptic membranes during the development of HS can be accompanied by qualitative changes of its metabolites, which bypass an transporter-molecule of EAAs. This circumstance can facilitate interaction of EAAs with receptors. Thus, it is believed that metabolic disturbances of PE metabolism in the synaptic membranes during the development of HS are a fundamental mechanism of neurotoxic damage to brain neurons. 


\section{Dysregulation of Phosphatidylserine Metabolism and Encephalopathy}

PS is an annular PL of opiate receptors and can bind opioid peptides. Given the fact that the capture of opioid peptides by receptors at action on the synaptic membranes of the PS-specific carboxylase is inhibited [51], it is possible that the PS depletion in the synaptic membranes of the frontal part of the brain and medulla oblongata in later stages of HS $[7,8]$ is accompanied by decrease in the coupling of endogenous peptides with the receptors. Data on the reduction of the content of the PS in the synaptic membranes during HS is consistent with the idea that cholinergic activation, which accompanies, in particular, massive blood loss, reduces the action of opioid peptides on neurons in the brain by reducing their specific binding to the cell membrane [52]. On the other hand, the opioid peptides are inhibitors of glutamate release through pathway of cellular signaling mediated by the activation of phospholipase A2 [53]. Thus, it is possible that synaptic membrane depletion in PS in the frontal part of the brain and medulla oblongata is a mechanism that increases the entrance of glutamate into the synaptic cleft and excessive accumulation of EAAs in extracellular fluid, as observed in the brain during prolonged hypotension [54].

It is known that PS has an important role in the mechanisms of activation of protein kinase $\mathrm{C}$, which due to ionic interactions with this PL, is connect with the plasma membrane and is remains in it [55]. Consequently, the PS depletion in synaptic membranes prevent an input in them of cytoplasmic protein kinase $\mathrm{C}$, weakening its activity in these sites of the neurons and, as a consequence, promotes increase of EAA excitotoxicity. Reduced level of PS in the synaptic membranes of the frontal part of the brain can be regarded as a key factor in the development of encephalopathy during HS because this PL has an important role in the mechanisms of memory and cognitive functions. Exogenous PS significantly improves memory, ability to training, the concentration of attention, and psycho-emotional condition $[3,56,57]$.

Thus it can be assumed that decreased level of PS in the synaptic membranes is a factor that strengthens the degenerative processes in the frontal part of the brain and in the medulla oblongata on later stages of HS, which associate with the activation of glutamatergic neurotoxic processes. These mechanisms presumably to have a particularly damaging effect on the neurons of the frontal lobe of the brain in late stages of HS, while compensatory recovery in PS levels occur in the medulla oblongata at this stage in the synaptic membranes $[7,8]$.

\section{Damaging Effects of Lysophosphatidylcholine}

Glutamatergic hyperactivation, accompanied by the stimulation of phospholipase $\mathrm{A} 2$ is presumably the main cause of the accumulation of an active metabolite of PC -
lyso-PC - in the synaptic membranes of the medulla oblongata in late stage of HS [7]. Accumulation lyso-PC can cause rapid entry of $\mathrm{Ca}^{2+}$ from the extracellular environment into the cytosol and increase of the mobilization of intracellular $\mathrm{Ca}^{2+}[58,59]$. Lyso-PC influences this process as through the stimulation of phosphorylation of phospholipase C [60], as well without the hydrolysis of phosphoinositides, at damaging of cell membranes. It is possible that the stimulating effect of lyso-PC on the activity of phospholipase $\mathrm{C}$ defines the normalization of PI level in the synaptic membranes of the medulla oblongata in later stage HS (7). However, it is known that lyso-PC dose-dependently inhibits PI kinase and PI 4-kinase [61]. Lyso-PC exhibits properties of inhibitor of cytidine triphosphate (CTP): cholinephosphat-cytidyltransferase, of enzyme, which possesses a high importance for the synthese of $\mathrm{PC}$ in the brain [47]. Moreover, lyso-PC is capable to stimulating of enzymes controlling the catabolism of PC, since it increases the activity of phospholipase $\mathrm{D}$ [62] and promotes the translocation of phospholipase A2 from the cytosol into the membrane [63]. Therefore, the accumulation of membrane lyso-PC can be considered among the causes of the depletion of PC in the synaptic membranes of the medulla oblongata on late stage of HS.

\section{Conclusion}

A reduction in the level of PI is a general feature of changes of the PLs composition of the synaptic membrane of the frontal lobe of the brain and the medulla oblongata during the initial stages of development of HS. This is appears to be largely determined by increased cholinergic, adrenergic, and glutamatergic stimulation. PI catabolism, in turn, initiates a series of activation mechanisms of neurotransmission that can be considered as compensatory-adaptive. Dysregulation of membrane PI metabolism is a crucial damaging pathway for neurotransmission in the frontal part of the brain during the late stages of HS. Decrease of PE decay throughout the development of HS and the depletion of PS in the late stages of HS are key mechanisms of damage of the synaptic membranes of the frontal lobes of the brain. This change of synaptic membranes structure mediates the activation of glutamatergic neurotoxic processes. Given the nootropic function of PS, a decrease in its level in the synaptic membranes during HS seems like a crucial mechanism of posthemorrhagic cognitive impairment. The depletion of PC and accumulation of lyso-PC in the synaptic membranes of the medulla oblongata can be considered as the main damaging factors of the brain showing the importance of the hyperactivity of the cholinergic nervous system in the pathogenesis of functional disorders CNS. Depletion of membrane PS and the accumulation of membrane PE in later stage of HS points at the characteristic features of PL-dependent initiation of glutamatergic hyperstimulation in the medulla oblongata.

Thus, the foregoing suggests that the dysregulation of PL metabolism in the synaptic membranes is a key mechanism 
of encephalopathy at HS. The normalization and stabilization of the PL composition of the neuronal membranes may be an important treatment of shock-induced disorders of brain functions.

\section{References}

[1] V. L. Kozhura, Zh.V. Solov'eva, I.S. Novoderzhkina, N.V. Nosova. "The neurochemical, molecular and ultrastructural mechanisms of the formation of latent postresuscitation encephalopathy". Anesteziol. Reanimatol. [in Russian]. nn. 5, pp. 52-56, May 1996

[2] V.V. Moroz. "Postreanimatological illness as dysregulation pathology." Dysregulation Pathology [in Russian], Moskow. 2002, pp. 233-259

[3] H.Y Kim., B.X Huang. A.A. Spector. "Phosphatidylserine in the brain: metabolism and function". Prog Lipid Res. vol. 56, pp. 1-18, November 2014

[4] M.C. Waugh. PIPs in neurological diseases. Biochem. Biophys. Acta, in press.

[5] J. Klein. "Membrane breakdown in acute and chronic neurodegeneration: focus on choline-containing phospholipids". J. Neural. Transm. vol.107, pp. 1027 1063, February 2000

[6] V.A. Tsirlin. "Bulbar vasomotor center - morpho functional and neurochemical Organization". Arterial Hypertension [in Russian].nn. 8, pp. 77- 81, August 2003

[7] G.F. Leskova. "Role of synaptic membranes phospholipids in regulation of neurotransmission mechanisms in Medula Oblangata during hemorrhagic shock in cats". Pathogenesis [in Russian]. nn. 4, pp. 57-65, April 2006

[8] G.F. Leskova. "Changes in phospholipid composition of synaptic membranes in frontal lobes of cerebral hemispheres in cats at various stages of hemorrhagic shock". Bull. Exp. Biol. Med. vol.146, pp. 401-404, April 2008

[9] H. Kubista, K. Kosenburger, P. Mahlknecht, H. Drobny, S. Boehm. "Inhibition of transmitter release from rat sympathetic neurons via presynaptic M1 muscarinic acetylcholine receptors". Br. J. Pharmacol. vol. 156, pp.1342-1352, April 2009

[10] T.G. Borda, G. Cremaschi, L. Sterin-Borda. "Haloperidol-mediated phosphoinositide hydrolysis via direct activation of alpha1-adrenoceptors in frontal cerebral rat cortex". Can. J. Physiol. Pharmacol. vol. 77, pp. 22-28, January 1999

[11] M.J. Berridge, M.D. Bootman, P. Lipp. "Calcium - a life and death signal”. Nature. vol. 395, pp. 645-648, October 1998

[12] S. Koizumi, P. Rosa, G.B. Willars. "Mechanisms underlying the neuronal calcium sensor-1-evoked enhancement of exocytosis in PC12 cell". J. Biol. Chem. vol. 277, pp. 30315-30324, May 2002

[13] Q. Zheng, J.A. Bobich, J. Vidugiriene. "Neuronal calcium sensor-1facilitates neuronal exocytosis through phosphatidylinositol 4-kinase". J. Neurochim. vol. 92, pp. 442-451, February 2005

[14] C.U.M. Smith Elements of molecular neurology. Atrium.
Chister. Wiley \& Sons. LTD. 2002.

[15] R.,Masgrau, J.M. Servitja, K.W.Yong, R. Pardo, E. Sarri, S.R. Nahorski, F. Picatoste. "Characterization of the metabotropic glutamate receptors mediating phospholipase $\mathrm{C}$ activation and calcium release in cerebellar granule cells: calcium-dependence of the phospholipase C response". Eur. J. Neurosci. vol. 13, pp. 248-256, January 2001

[16] A.A. Boldyrev. "Functional interaction between various glutamate receptors". Bull. Exp. Biol. Med. [in Russian]. vol. 130, pp. 244-251, September 2000

[17] I.I. Abramez, I.B. Komissarova. "Glutamatergic mechanisms of brain Ischemic damages". J. AMN Ukraina [in Russian]. nn. 7, pp. 613-633, July 2001

[18] P.V. Avdonin. "The structura and signal properties of G-protein-coupled receptors complexes". Biol. Membr. [in Russian]. vol. 22, pp. 3-26, January 2005

[19] V.A.Tkachuk. "Molecular mechanisms of neuroendocrine regulation". Soros. Educational J. [in Russian]. nn. 6, pp. 16-20, June 1998

[20] C.P. Morgan, A. Skippen, B. Segui, A. Ball, V. Allen-Baume, B. Larijani, J., Murray-Rust, N. McDonald, G. Sapkota, N. Morrice, S. Cockcroft. "Phosphorylation of a distinct structural form of phosphatidylinositol transfer protein alpha at Ser16 by protein kinase $\mathrm{C}$ disrupts receptor-mediated phospholipase $\mathrm{C}$ signaling by inhibiting deliver of phosphatidylinositol to membranes". J. Biol. Chem. vol. 279, pp. 47159-47171, November 2004

[21] S.M. Baijalieh, R.H. Scheller. "The biochemistry of neurotransmitter secretion". J. Biol. Chem. vol. 270, pp. 1971-1974, February 1995

[22] S.K. DebBurman, J. Ptasienski, E. E. Boetticher, J.W. Lomasney, J.L.Benovic, M.M. Hosey. "Lipid-mediated regulation of $\mathrm{G}$ protein-coupled receptor kinases 2 and 3. J. Biol. Chem. vol. 270, pp. 5742-5747, March 1995

[23] K.W. Young, D. Billups, C.P. Nelson, N. Johnston, J.M. Willets, M.J. Schell, R.A. Challiss, S.R. Nahorski. "Muscarinic acetylcholine receptor activation enhances hippocampal neuron excitability and potentiates synaptically evoked $\mathrm{Ca}(2+)$ signals via phosphatidylinositol 4,5-bisphosphate depletion". Mol. Cell. Neurosci. vol, 30, pp. 48-57, September 2005

[24] S.J. Veerasingham, M.,Yamazato, K.H. Berecek, J.M. Wyss, M.K. Raizada. "Increased PI3-kinase in presympathetic brain areas of the spontaneously hypertensive rat". Circ. Res. vol. 96 , pp. 277-279, January 2005

[25] T.T. Ching, D.S. Wang, A.L. Hsu, P.J. Lu, C.S. Chen. "Identification of multiple phosphoinositide-specific phospholipases D as new regulatory enzymes for phosphatidylinositol 3,4, 5-trisphosphate". J. Biol. Chem. vol. 274, pp. 8611-8617, March 1999

[26] Y. Sang, D. Cui, X. Wang. "Phospholipase D and phosphatidic acid-mediated generation of superoxide in Arabidopsis". Plant Physiol. vol.126, pp. 449-458, August 2001

[27] S.T. Kim, Y.H. Chung, H.S. Lee, S.J. Chung, J.H. Lee, U.D. Sohn, Y.K. Shin, E.S. Park, H.C. Kim, J.H. Jeong. "Protective effects of phosphatidylcholine on oxaliplatin-induced neuropathy in rats. Life Sci., in press. 
[28] T. Hirabayashi, T. Murayama, T. Shimiza. "Regulatory mechanism and physiological role of cytosolic phospholipase A2." Biol. Pharmacol. Bull. vol. 27, pp. 1168-1173, August 2004

[29] G. Massicotte. "Modification of glutamate receptors by phospholipase A2: its role in adaptive neural plasticity." Cell. Mol. Life Sci. vol. 57, pp. 1542-1550, October 2000

[30] F. St-Gelas, C. Menard, P. Congar, L.E. Trudeau, G. Massicotte. Postsynaptic injection of calcium-independent phospholipase A2 inhibitors selectively increases AMPA receptor-mediated synaptic transmission. Hippocampus. vol.14, pp. 319-325, January 2004

[31] H. Nishio, T. Takeuchi, F. Hata, O. Yagasaki. " $\mathrm{Ca}(2+)$-independent fusion of synaptic vesicles with phospholipase A2-treated presynaptic membranes in vitro". Biochem. J. vol. 318 (3), pp. 981-987, September 1996

[32] M.A. DeCoster, G. Lambeau, M. Lazdunski, N.G. Bazan. "Secreted phospholipase A2 potentiates glutamate-induced calcium increase and cell death in primary neuronal cultures". J. Neurosci. Res. vol. 67, pp. 634-645, March 2002

[33] A.L. Taylor, S.J. Hewett. "Potassium-evoked glutamate release liberates arachidonic acid from cortical neurons". J. Biol. Chem. vol. 277, pp. 43881-43887, November 1992

[34] Q. Chen, D.P. Li, H.L. Pan. "Presynaptic alpha1 adrenergic receptors differentially regulate synaptic glutamate and GABA release to hypothalamic presympathetic neurons". J. Pharmacol. Exp. Ther. vol. 316, pp. 733-742, 2006

[35] J.D., Navarro-Lopez, J.M. Delgado-Garcia, J. Yajeya. "Cooperative glutamatergic and cholinergic mechanisms generate short-term modifications of synaptic effectiveness in prepositus hypoglossi neurons". J. Neurosci. vol. 25, pp. 9902-9906, February 2005

[36] F. Facchinetti, N.J. Hack, R. Balázs. "Calcium influx via ionotropic glutamate receptors causes long lasting inhibition of metabotropic glutamate receptor-coupled phosphoinositide hydrolysis". Neurochem. Int. vol. 33, pp. 263-270, September 1998

[37] F.E. Sieber, R.J. Traystman, L.J. Martin. "Delayed neuronal death after global incomplete ischemia in dogs is accompanied by changes in phospholipase C protein expression". J. Cereb. Blood Flow. Metab. vol.17, 527- 533, May 1997

[38] M.M. http://www.ncbi.nlm.nih.gov/pubmed/2201284Billah, J.C. Anthes. "The regulation and cellular functions of phosphatidylcholine hydrolysis". Biochem J. vol. 269, pp. 281-291, July 1990

[39] S. Llahi, J.N. Fain. "Alpha 1-adrenergic receptor mediated activation of phospholipase D in rat cerebral cortex". J. Biol. Chem. vol.. 267, pp. 3679-3685, February 1992

[40] N.G. Bazan, B. Tu, E.B. Rodriguez de Turco. "What synaptic lipid signaling tells us about seizure-induced damage and epileptogenesis”. Prog. Brain Res. vol. 135, pp. 175-185, 2002

[41] W.B. Bollag, X. Zhong, M.E. Dodd, D.M. Hardy, X. Zheng, W.T. Allred. "Phospholipased signaling and extracellular signal-regulated kinase-1 and -2 phosphorylation (activation) are required for maximal phorbol ester-induced transglutaminase activity, a marker of keratinocyte differentiation". J. Pharmacol. Exp. Ther. vol. 312, pp.1223-1231, March 2005
[42] Min D.S., Park S.K., Exton J.H. "Characterization of a rat brain phospholipase D isozyme”. J. Biol. Chem. vol. 273, pp. 7044-7051, March 1998

[43] M.R Castillo, J.R. Babson. "Ca(2+)-dependent mechanisms of cell injury in cultured cortical neurons". Neurosci. vol. 86, pp. 1133-1144, October 1998

[44] M. McDermontti, M.J. Wakelam, A.J. Morris. "Phospholipase D”. Biochem. Cell. Biol. vol. 82, pp. 225-253, February 2004

[45] M. Liscovitch, V., Chalifa, P. Pertile, C.S. Chen, L.C Cantley."Novel function of phosphatidylinositol 4, 5-bisphosphate as a cofactor for brain membrane phospholipase D". J. Biol. Chem. vol. 269, pp. 21403-21406, August 1994

[46] T. Gasull, E. Sarri., N. Degregorio-Rocasolano, R. Trullas. "NMDA receptor overactivation inhibits phospholipid synthesis by decreasing choline ethanolamine phosphotransferase activity". J. Neurosci. vol. 23, pp. 4100-4107, May 2003

[47] R.M. Adibhatla, J.F. Hatcher, R.J. Dempsey. "Cytidine-5'-diphosphocholine affects CTP phosphocholine cytidylyltransferase and lyso phosphatidylcholine after transient brain ischemia". J. Neurosci. Res. vol. 76, pp. 390-396, May 2004

[48] V. L. Kozhura. "Neurobiological mechanisms of massive blood loss". Anaestheziol. Reanimatol. [in Russian]. nn. 6, pp. 51-53javascript:babSpeakIt('английский',170574);, June 2001

[49] S. Persard, V. Panagia. "Abnormal synthesis of N-methylated phospholipids during calcium paradox of the heart". J. Mol. Cell. Cardiol. vol. 27, pp. 579-587, 1995

[50] A.A. Vartanyan, G.V. Apikyan, V.F Vanyushin. "Methylation of phospholipids and synaptic capture of mediator amino acids". Izv. AN USSR [in Russian], ser. Biol., nn. 5, pp. 786-789, May November 1990

[51] O.S. Belokoneva, O.V. Zaycev. "Role of membrane lipids in regulation of neuromediators receptors functioning". Biochemistry [in Russian]. vol. 58, pp. 1685-1708, November 1993

[52] [52] V.P. Fisenko. "Neurochemical laws of opioid analgesics action on cortex. Bull. Exp. Biol. Med. [in Russian]. vol. 132, pp. 4-11, July 2001

[53] W. Zhu Z.Z. Pan. "Mu-opioid-mediated inhibition of glutamate synaptic transmission in rat central amygdala neurons". Neuroscience. vol.133, pp. 97-103, 2005

[54] V. L. Kozhura, N.V. Nosova. "Apoptosis as the mechanism of delayed posthypoxic encephalopathy". Bull. Exp. Biol. Med. [in Russian]. appendix 2, pp. 30-32, 2000

[55] D.M. Faller, D.M. Schilde. "Molecular Biology of Cell". [in Russian]. Moskow. 2003.

[56] M.A. McDaniel, S.F. Maier, G.O. Einstein. "Brain-specific" nutrients: a memory cure?" Nutrition. vol. 19, pp. 957-975, November - December 2003

[57] M. Kingsley. "Effects of phosphatidylserine supplementation on exercising Humans". Sports Med. vol. 36, pp. 657-669, 2006 
[58] J.T. Wong, K. Tran, G.N. Pierce, A.C. Chan, K. O, P.C. Choy. "Lysophosphatidylcholine stimulates the release of arachidonic acid in human endothelial cells". J. Biol Chem. vol. 273, pp. 6830-6836, March 1998

[59] H. Ozaka, K. Ishii, H. Arai, N. Kume, T. Kita. "Lysophosphatidylcholine activates mitogen-activated protein kinases by a tyrosine kinase-dependent pathway in bovine aortic endothelial cells". Atherosclerosis. vol. 143, pp. 261-266, April 1999

[60] B.V., Bassa, D.D. Roh, N.D. Varzirl, M.A. Kirschenbaum, V.S Kamanna. "Lysophosphatidylcholine activates mesangial cell PKC and MAP kinase by PLCgamma-1 and tyrosine kinase-Ras pathways". Am. J. Physiol. Renal. Physiol. vol. 277 (2), pp. F328-F337, September 1999
[61] S.Y. Liu, C.H. Yu, J.A. Hays, V. Panagia, N.S. Dhalla. "Modification of heart sarcolemmal phosphoinositide pathway by lysophosphatidylcholine". Biochim. Biophys. Acta. vol.1349, pp. 264-274, November 1997

[62] D.A. Cox, M.L. Cohen. "Lysophosphatidylcholine stimulates phospholipase D in human coronary endothelial cells: role of PKC”. Am. J. Phisiol. vol. 271 (2), pp. H1706-H1710, October 1996

[63] L.S. Golfman, N.J. Haughey, J.T. Wong, J.Y. Jiang, D. Lee, J.D. Geiger, P.C. Choy. "Lysophosphatidylcholine induces arachidonic acid release and calcium overload in cardiac myoblastic H9c2 cells”. J. Lipid. Res. vol. 40, pp. 1818-1826, October 1999. 